\title{
Almost Hamiltonian Groups
}

\author{
Tuval Foguel Pantelimon Stănic $\breve{A}^{*}$
}

\begin{abstract}
In this paper we investigate the following problem in Group Theory: which properties $\mathcal{P}$ transfer (or do not transfer) from all cyclic subgroups, or all abelian subgroups to all arbitrary subgroups? We solve this problem completely when $\mathcal{P}$ is the property of having finite index in its normal closure, proving that $\mathcal{P}$ carries from abelian, but not from cyclic to arbitrary subgroups. We use primarily some results of B.H. Neumann.
\end{abstract}

\section{Preliminaries}

In this section, we review some standard concepts from group theory, and establish some conventions concerning notation. Let $G$ be a group, and $Z=Z(G)$ be its center. If $H$ is a subgroup in $G$, then $|G: H|$ denotes its index in $G$, and $H^{G}$ the normal closure in $G$, that is, the intersection of all normal subgroups of $G$ containing $H$. Denote by $[x, y]:=x^{-1} y^{-1} x y$, where $x, y \in G$, $x^{y}:=y^{-1} x y$. Let $G^{\prime}=[G, G]$ be the derived subgroup of $G$. If $S$ is a subset of $G$, then $|S|$ denotes the cardinal of $S$, and $\langle S\rangle$ the subgroup generated by $S$ in $G$. For a group $G$ and a subset $S \subseteq G$, we define the normalizer $N_{G}(S)=\left\{g \in G \mid S^{g}=S\right\}$ and $\left|S^{G}\right|=\left|G: N_{G}(S)\right|$. Also, $C_{G}(S)=\left\{g \in G \mid x=x^{g}, \forall x \in S\right\}$ is the centralizer of $S$. We define the core $H_{G}$ as the largest normal subgroup of $G$ contained in $H$. For more definitions we refer the reader to [6].

Many authors have investigated the structure of groups having finite index in their normal closure. Groups in which all subgroups are normal-by-finite (that is, the core $H_{G}$ of $H$ in $G$ has finite index in $\left.H,\left|H: H_{G}\right|<\infty\right)$ are studied in [1,2,3,7], where in particular it is proved that if $\mathrm{G}$ is a group with that property, and all periodic homomorphic images of $\mathrm{G}$ are locally finite, then $\mathrm{G}$ is abelian-by-finite. In [2] the authors consider groups satisfying the minimal condition on subgroups which are not normal-by-finite.

In spite of the fact that more of the machinery was existent, apparently no one investigated (explicitly) the properties of a group that carry from abelian (or cyclic) to arbitrary subgroups. In

\footnotetext{
*Associated with the Institute of Mathematics "Simion Stoilow" of The Romanian Academy, Bucharest, Romania

${ }^{1} 2000$ Mathematics Subject Classification: 20D35, 20F22, 20F24

${ }^{2}$ Keywords: Infinite groups; Normality; Pronormality; Finite index subgroups.
} 
this note we propose this general problem for future research and prove that the property of having finite index in its normal closure carries from abelian, but not from cyclic to arbitrary subgroups.

\section{The Results}

A subgroup property $\mathcal{P}$ is closed with respect to joins of cyclic subgroups, if given any set of cyclic subgroups with property $\mathcal{P}$, say $\left\{H_{\lambda}: \lambda \in \Lambda\right\}$, then $\left\langle H_{\lambda}: \lambda \in \Lambda\right\rangle \in \mathcal{P}$.

Definition Given a group $G$ and a subgroup property $\mathcal{P}$ that is closed with respect to joins of cyclic subgroups, we say that $G$ is a $\mathcal{P}$-Hamiltonian group if every subgroup of $G$ has property $\mathcal{P}$. In the case that $\mathcal{P}=$ normality, we call $G$ a Hamiltonian group.

Theorem 2.1. Let $\mathcal{P}$ be a subgroup property closed with respect to joins of cyclic subgroups. Then, the following statements are equivalent:

(i) $G$ is a $\mathcal{P}$-Hamiltonian group,

(ii) All abelian subgroups of $G$ have property $\mathcal{P}$,

(iii) All cyclic subgroups of $G$ have property $\mathcal{P}$.

Proof. It is immediate that $(i) \Rightarrow(i i) \Rightarrow(i i i)$. We will show that $(i i i) \Rightarrow(i)$. If all cyclic subgroups of $G$ have property $\mathcal{P}$, since property $\mathcal{P}$ is closed with respect to joins, given $H \leq G$

$$
H=\langle\langle h\rangle: h \in H\rangle
$$

so $H$ has property $\mathcal{P}$. Thus $G$ is a $\mathcal{P}$-Hamiltonian group.

Definition Let $G$ be a group and $\mathcal{P}$ be a subgroup property in $G$ that is closed with respect to joins of cyclic subgroups.

1. We say that $G$ is a $A P H 1$ group if every subgroup of $G$ has finite index in a subgroup with property $\mathcal{P}$.

2. We say that $G$ is a $A P H 2$ group if every abelian subgroup of $G$ has finite index in a subgroup with property $\mathcal{P}$.

3. We say that $G$ is a $A P H 3$ group if every cyclic subgroup of $G$ has finite index in a subgroup with property $\mathcal{P}$. 
In the case that $\mathcal{P}=$ normality we call $G$ an $A H i$-group where $i=1,2$, or $3(A P H$ stands for almost $\mathcal{P}$-Hamiltonian). We use $[X]$ to denote the class of groups having property $X$. It is worth mentioning that $[A P H 1] \subseteq[A P H 2] \subseteq[A P H 3]$, always.

Example 2.2. It is easy to see that if $\mathcal{P}=$ subgroup, then

$$
[A P H 1]=[A P H 2]=[A P H 3]
$$

since every group is a subgroup-Hamiltonian group.

Example 2.3. If $\mathcal{P}=$ group, then only $\langle 1\rangle \in[P H]$, so a group $G \in[A P H 1],[A P H 2]$, or $[A P H 3]$ if and only if every cyclic subgroup has finite index in $G$, that is, $G$ is finite, so

$$
[A P H 1]=[A P H 2]=[A P H 3] .
$$

In [5], B.H. Neumann investigated interrelations of various classes of groups defined by certain properties. We shall recall some definitions here:

$F C$ : the classes of conjugate elements of $G$ are finite, that is, $\left|G: C_{G}(\langle g\rangle)\right|<\infty$.

$F D: G^{\prime}$ is finite.

$F I Z$ : the center has finite index.

$A H 1$ : if $H \leq G$, then $\left[H^{G}: H\right]<\infty$ (which Neumann calls $Y$ ).

$A H 3$ : if $\langle g\rangle \leq G$, then $\left[\langle g\rangle^{G}:\langle g\rangle\right]<\infty$ (which Neumann looks at in Lemma $3.6[5]$ ).

$F G: G$ is finitely generated.

If we strengthen "finite" to "boundedly finite" we write for the corresponding properties $B F C$, etc.

An interesting question is to discuss the relationship between the three cases of almost $\mathcal{P}$ Hamiltonian groups for different properties $\mathcal{P}$ that are closed under joins of cyclic subgroups like quasinormality, etc. One cannot stop from wondering whether other properties carry from abelian (or even cyclic) to arbitrary subgroups.

Another interesting question to investigate is when a property is closed under joins of cyclic subgroups for certain family of groups. For example, from Theorem 2.6 and Corollary 2.9 of [4], pronormality is closed under joins of cyclic subgroups when $G$ is a locally solvable FC-group, or a solvable polycyclic-by-finite group.

The following result summarizes much of the work of [5]. 
Theorem 2.4. The following relations are true:

$$
\begin{aligned}
& {[F G] \cap[F D]=[F G] \cap[A H 1]=[F G] \cap[A H 2]=[F G] \cap[A H 3]=[F G] \cap[F C],} \\
& {[F D]=[A H 1]}
\end{aligned}
$$

Proof. It is known that $[A H 1] \subseteq[A H 2] \subseteq[A H 3]$, which implies that

$$
[F G] \cap[A H 1] \subseteq[F G] \cap[A H 2] \subseteq[F G] \cap[A H 3]
$$

We infer from Lemma 3.6 of $[5]$ that $[A H 3] \subseteq[F C]$, and from Corollary 3.8 that $[F G] \cap[A H 1]=$ $[F G] \cap[F C]$. Also, from Theorem 13.1 of $[5]$, one gets $[A H 1]=[F D]$. Putting these facts together we get

$$
[F G] \cap[A H 1] \subseteq[F G] \cap[A H 2] \subseteq[F G] \cap[A H 3] \subseteq[F G] \cap[F C]=[F G] \cap[A H 1]=[F G] \cap[F D]
$$

Thus the proof of the theorem.

The main result of this paper is the next theorem.

Theorem 2.5. The following relations are true:

$$
\begin{aligned}
& {[A H 1]=[A H 2],} \\
& {[A H 3]=[F C],} \\
& {[A H 1] \varsubsetneqq[A H 3] .}
\end{aligned}
$$

\section{The Proof}

The proof of the previous theorem will follow by putting together the following three lemmas.

Lemma 3.1. $[A H 3]=[F C]$

Proof. Lemma 3.6 of $[5]$ implies that $[A H 3] \subseteq[F C]$. It suffices to show the other inclusion. Let $G \in[F C]$, and $g$ an element of $G$. Thus, the classes of conjugate elements of $G$ are finite, in particular

$$
g^{G}=\left\{a_{1}^{-1} g a_{1}, \ldots, a_{n}^{-1} g a_{n}\right\},
$$

for some $a_{1}, \ldots, a_{n} \in G$. Consider the subgroup of $G$, say $H$, generated by $\left\{a_{1}, a_{2}, \ldots, a_{n}, g\right\}$, which is certainly finitely generated. By Theorem 2.4 we know that

$$
[F C] \cap[F G]=[F D] \cap[F G]=[A H 1] \cap[F G] .
$$

Using (6) and the fact that $\langle g\rangle^{H}=\langle g\rangle^{G}=H$, we obtain $\left|\langle g\rangle^{H}:\langle g\rangle\right|=\left|\langle g\rangle^{G}:\langle g\rangle\right|<\infty$, which proves that $G \in[A H 3]$. 
Lemma 3.2. $[A H 1]=[A H 2]$

Proof. Suppose that $G \in[A H 3]-[A H 1]$. By [5] and Lemma 3.1, $G \in[F C]-[F D]$. By Lemma 11.1 of $[5]$ if $G \in[F C]-[F D]$, then $G$ has a subgroup

$$
G^{1}=\left\langle a_{1}, b_{1}, c_{1}, a_{2}, b_{2}, c_{2}, \ldots:\left[a_{i}, b_{i}\right]=c_{i} \neq c_{j} \text { for } i \neq j,\left[a_{i}, a_{j}\right]=\left[a_{i}, b_{j}\right]=\left[b_{i}, b_{j}\right]=1\right\rangle .
$$

Case 1:

$$
\left[a_{i}, c_{i}\right] \neq 1 \text { or }\left[b_{i}, c_{i}\right] \neq 1
$$

for infinitely many $i$. Without loss of generality we may assume that

$$
\left[a_{i}, c_{i}\right] \neq 1 \text { or }\left[b_{i}, c_{i}\right] \neq 1
$$

for all $i$.

Define now a class $[K]$ of groups generated by $\left\{a_{i}, b_{i}, c_{i}\right\}_{i \in \mathbb{N}}$ subject to the relations

$$
\left[a_{i}, b_{i}\right]=c_{i} \neq 1,\left[a_{i}, a_{j}\right]=\left[a_{i}, b_{j}\right]=\left[b_{i}, b_{j}\right]=1, \text { if } i \neq j,
$$

each subgroup $N_{i}=\left\langle a_{i}, b_{i}\right\rangle\left(=\left\langle a_{i}, b_{i}, c_{i}\right\rangle\right) \in[F C]$ and $N_{i} \cap N_{j}=\{1\}$ if $i \neq j$. By Lemma 11.3 of [5], we obtain that $G^{1} / Z\left(G^{1}\right)=G^{*}$ is a $K$-group. We will denote the $\operatorname{coset} a_{i} G^{1}$ by $a_{i}^{*}$. Now by Lemmas 10.2 and 10.5 of [5], $A^{*}=\left\langle a_{i}^{*}: i \in \mathbb{N}\right\rangle$ has infinitely many distinct conjugates. Thus, $A=\left\langle a_{i}: i \in \mathbb{N}\right\rangle$ has infinitely many distinct conjugates, and $A$ is also abelian. So $G \notin[A H 2]$.

Case 2:

$$
\left[a_{i}, c_{i}\right] \neq 1 \text { or }\left[b_{i}, c_{i}\right] \neq 1
$$

for a finite number of indices $i$. Without loss of generality we may assume that

$$
\left[a_{i}, c_{i}\right]=\left[b_{i}, c_{i}\right]=1
$$

for all $i$. Note that in this case $G$ is nilpotent of class 2. So by Lemmas 8.3, 9.2, 11.6 and 11.7 of [5], $G$ has an abelian subgroup with infinitely many distinct conjugates, thus $G \notin[A H 2]$.

Thus if $G \in[A H 3]-[A H 1]$, then $G \in[A H 3]-[A H 2]$, so $[A H 1]=[A H 2]$.

Lemma 3.3. $[A H 1] \varsubsetneqq[A H 3]$

Proof. Let $G$ be the restricted direct product of infinitely many quaternion groups (i.e. $G \leq \prod_{\lambda} Q_{\lambda}$ where each $Q_{\lambda}$ is isomorphic to quaternion group and if $g \in G$, then $g=\prod_{\lambda} q_{\lambda}$ where $q_{\lambda} \in Q_{\lambda}$ and all but a finite number of $q_{\lambda}$ 's are the identity). Certainly, $G \in[A H 3]$. Now, we show that $G \notin[A H 1]$. Clearly, using Neumann [5] notation, $G \in[I I I] \subseteq[F C]-[F D]=[A H 3]-[A H 1]$, Lemma 3.1 and Theorem 2.4. 


\section{References}

[1] J. Buckley, J. C. Lennox, B. H. Neumann, H. Smith, and J. Wiegold, Groups with all subgroups normal-by-finite, J. Austral. Math. Soc. Ser. A 59 (1995), 384-398.

[2] S. Franciosi and F. de Giovanni, Groups with many normal-by-finite subgroups, Proc. AMS 125 (1997), 323-327.

[3] S. Franciosi, F. de Giovanni, and M. L. Newell, Groups whose subnormal subgroups are normal-by-finite, Comm. Algebra 23 (1995), 5483-5497.

[4] F. de Giovanni and G. Vincenzi, Pronormality in infinite groups, Math. Proc. Royal Irish Acad. 100 (2000), 189-203.

[5] B. H. Neumann, Groups with finite classes of conjugate subgroups, Math. Zeit. 63 (1955), $76-96$.

[6] D. J. S. Robinson, A course in the theory of groups (2nd ed.), Graduate Texts in Mathematics 80, Springer-Verlag, New York, 1996.

[7] H. Smith and J. Wiegold, Locally graded groups with all subgroups normal-by-finite, J. Austral. Math. Soc. Ser. A 60 (1996), 222-227.

[8] N.F. Kuzenny $\breve{1}$ and I.Ya. Subbotin, Groups in which all of the subgroups are pronormal (Russian), Ukrain. Mat. Zh. 39 (1987), 325-329, 405.

[9] I.Ya. Subbotin and N.F. Kuzenny $\breve{1}$, Locally solvable groups in which all infinite subgroups are pronormal (Russian), Izv. Vyssh. Uchebn. Zaved. Mat. 1988, 77-79; Transl. in Soviet Math. (Iz. VUZ) 32 (1988), 126-131.

\section{Affiliation:}

(T.F. \& P.S): Auburn University Montgomery, Department of Mathematics, P.O. Box 244023, Montgomery, AL 36124-4023, USA; \{tfoguel, pstanica\}@mail.aum.edu 